\title{
Stability of the higher-bound states in a saturable self-focusing medium
}

\author{
J. M. Soto-Crespo, D. R. Heatley, and E. M. Wright* \\ Optical Sciences Center, University of Arizona, Tucson, Arizona 85721 \\ N. N. Akhmediev \\ Institute of Physical Problems, Moscow 103460, U.S.S.R.
}

(Received 2 November 1990)

\begin{abstract}
We present an investigation of the stability of the cylindrically symmetric higher-bound states that can be formed in a self-focusing medium with saturation. The higher-bound states are found to display transverse instabilities that break the azimuthal symmetry of the system. An approximate analytic theory is presented that correctly describes the qualitative variations of the numerically calculated instability growth rates.
\end{abstract}

\section{INTRODUCTION}

The problem of the self-trapping of optical beams $[1,2]$ in nonlinear media has been studied extensively over the last three decades both experimentally [3] and theoretically [4]. However, most interest in this field has centered on the lowest-bound state (or ground state) that is cylindrically symmetric and has no field nodes (zero crossings). Yankauskas [5] first showed that, for a medium with a Kerr-type nonlinearity, there exists an infinite number of bound states with cylindrical symmetry, each being characterized by the number of field nodes, and a similar conclusion was reached by Haus [6] on the basis of numerical solutions. The $n$th bound state has a central bright spot surrounded by $n$ annular rings of varying size. From the theoretical point of view the stability of these nonlinear waves is of great interest. Vakhitov and Kolokolov [7] first obtained stability conditions for the ground state. These have been shown to apply to a wide range of nonlinearities, both with and without saturation [8]. In contrast, to the best of our knowledge, only one paper by Kolokolov and Sykov [9] has considered the stability of the higher-bound states, and their findings have never been tested by direct numerical simulations of the nonlinear wave equation.

In this paper, we present an investigation of the stability of the cylindrically symmetric higher-bound states that can exist in a self-focusing medium with saturation. For this case the ground state is known to be stable, but we find that the higher-bound states display transverse (modulation) instabilities which break the azimuthal symmetry of the system [8]. Transverse instabilities arise for nonlinear waves whose description requires fewer dimensions than that of the coordinate space. For the case at hand, the higher-bound states can be described using the coordinate system $(r, z)$, which is two dimensional. The transverse instability corresponds to growth of perturbation eigenmodes which depend on the azimuthal angular variable $\theta$ in three-dimensional cylindrical coordinates. Mathematically speaking, the bound-state solutions can be viewed as saddle points in the phase space of our system. It is then straightforward to show that the real and imaginary parts of each unstable perturbation eigenmode represent the outgoing trajectories from these saddle points, whereas the complex conjugates of these represent the trajectories that are moving into the saddle-type point. Here we use numerical methods to determine the unstable perturbation eigenmodes and corresponding growth rates which result from linearizing the nonlinear wave equation, and an approximate analytic theory is presented which correctly describes the qualitative variations of the numerically calculated instability growth rates. We also present direct numerical simulations of the nonlinear wave equation which corroborate our findings, some of which are at variance with those of Kolokolov and Sykov [9]. These simulations show that upon propagation the annular rings of the higher-bound modes break into a series of regularly spaced filaments or hot spots, the number of which is accurately predicted by the numerical and analytic stability results. Such periodic beam breakup into filaments has previously been studied both experimentally [10-13] and theoretically [14-16] for input fields other than bound states. In particular, for the case of a Kerr-type nonlinearity, Campillo, Shapiro $[11,12]$, and Suydam [14] have stressed the fact that periodic beam breakup results from a transverse instability.

The remainder of this paper is organized as follows. Section II describes the nonlinear wave equation and notation we have employed and discusses the stationary bound-state solutions. The stability of the bound-state solutions is investigated in Sec. III both numerically and using an approximate analytic model. Direct numerical simulations of the nonlinear wave equation which corroborate the results from the stability analyses are given in Sec. III D. Finally Sec. IV contains our summary and conclusions. 


\section{BASIC THEORY \\ AND STATIONARY SOLUTIONS}

In this section we describe the nonlinear wave equation and notation used in this paper, and discuss the stationary solutions of the problem

\section{A. Nonlinear wave equation}

We consider the propagation of a monochromatic, linearly polarized electric field of frequency $\omega$ in a saturable self-focusing medium characterized by the intensitydependent dielectric constant [17]

$$
\epsilon\left(|E|^{2}\right)=\epsilon_{b}+\Delta \epsilon_{\text {sat }} \frac{|E|^{2}}{\left(1+|E|^{2}\right)},
$$

where $\epsilon_{b}$ is the background dielectric constant, $\Delta \epsilon_{\text {sat }}>0$ is the saturated change in dielectric constant, and $|E|^{2}$ is scaled such that it is the intensity in units of the saturation intensity. Then writing the positive frequency component of the electric field $E$ in the form

$$
E=A(X, Y, Z) \exp \left(i k_{0} n_{0} Z\right),
$$

where $k_{0}=\omega / c$, and $n_{0}$ is the effective refractive index, we obtain the following quasi-optical equation for the slowly varying envelope $A[17]$ :

$$
\begin{aligned}
2 i k_{0} n_{0} \frac{\partial A}{\partial Z}+\frac{\partial^{2} A}{\partial X^{2}}+\frac{\partial^{2} A}{\partial Y^{2}} & -k_{0}^{2}\left(n_{0}^{2}-\epsilon_{b}\right) A \\
& +k_{0}^{2} \Delta \epsilon_{\mathrm{sat}} \frac{|A|^{2} A}{1+|A|^{2}}=0
\end{aligned}
$$

We now write this equation in dimensionless form by introducing the scaled variables defined by

$$
\begin{aligned}
& x=k_{0} X\left(\Delta \epsilon_{\text {sat }}\right)^{1 / 2}, \quad y=k_{0} Y\left(\Delta \epsilon_{\text {sat }}\right)^{1 / 2}, \\
& z=k_{0} Z \Delta \epsilon_{\text {sat }} / 2 n_{0},
\end{aligned}
$$

in terms of which the nonlinear wave equation becomes

$$
i \frac{\partial A}{\partial z}+\nabla_{T}^{2} A-q^{2} A+\frac{|A|^{2} A}{1+|A|^{2}}=0 .
$$

Here $q^{2}=\left(n_{0}^{2}-\epsilon_{b}\right) / \Delta \epsilon_{\text {sat }}$ with $q$ playing the role of a scaled effective index for the nonlinear wave, and the transverse Laplacian describing beam diffraction in cylindrical geometry is

$$
\nabla_{T}^{2}=\nabla_{r r}^{2}+\frac{1}{r^{2}} \frac{\partial^{2}}{\partial \theta^{2}}
$$

with

$$
\nabla_{r r}^{2}=\frac{\partial^{2}}{\partial r^{2}} \frac{+1}{r} \frac{\partial}{\partial r} .
$$

Equation (5) is the starting point for our discussion of propagation in a saturable self-focusing medium.

\section{B. Stationary bound states}

The stationary solutions are defined by setting $\partial A / \partial z=0$. If we concentrate on those stationary solutions with cylindrical symmetry $A=A(r)$, and assume $A$

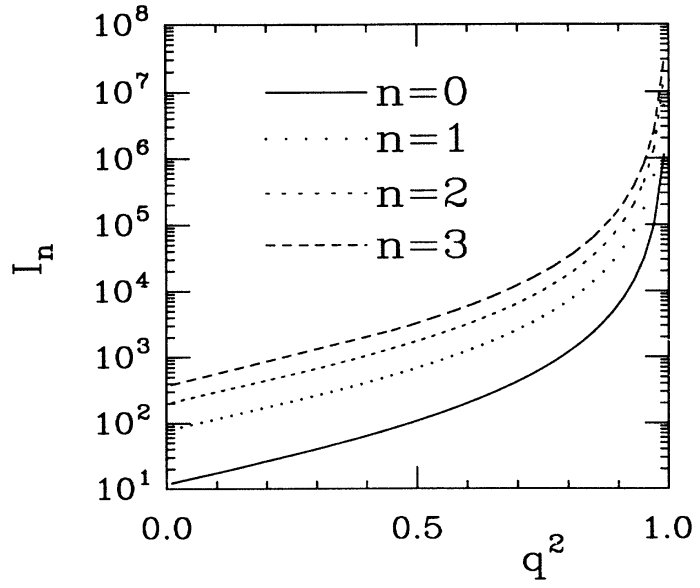

FIG. 1. Energy flux vs $q^{2}$ for the first four stationary bound states.

is real without loss of generality, then Eq. (5) becomes

$$
\nabla_{r r}^{2} A-q^{2} A+\frac{A^{3}}{1+A^{2}}=0
$$

with the boundary conditions

$$
\left.\frac{\partial A}{\partial r}\right|_{r=0}=\left.\frac{\partial A}{\partial r}\right|_{r \rightarrow \infty}=0,\left.\quad A(r)\right|_{r \rightarrow \infty}=0 .
$$

It is straightforward to show that $0 \leq q^{2} \leq 1$ [7]. Then for each allowed value of $q^{2}$, Eq. (7) has bound-state solutions that we designate as $A_{n}, n=0,1,2 \ldots$, the $n$th solution having $n$ field nodes. We do not here address the question of the existence or uniqueness of these solutions.

In general the bound states of Eq. (7a) cannot be solved in closed analytical form and must be found numerically. We have obtained numerical solutions of Eqs. (7) using a shooting method, and for a given value of $q^{2}$ the corre-

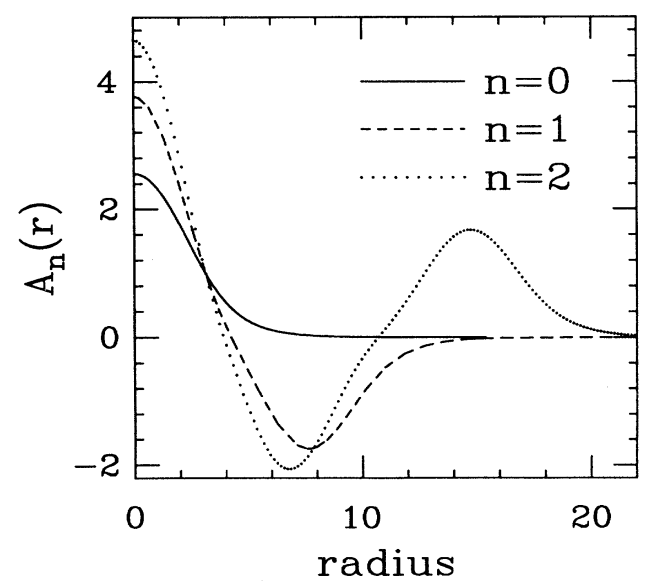

FIG. 2. Field profiles of the bound states corresponding to $q^{2}=0.5$ and $n=0$ (solid line), $n=1$ (dashed line), and $n=2$ (dotted line). 
sponding bound-state field profiles $A_{n}(r)$ were obtained. From these numerical solutions we then calculated the self-trapped energy flux of the $n$th bound-state solution as

$$
I_{n}=\int_{0}^{\infty} 2 \pi r d r A_{n}^{2}(r)
$$

Figure 1 shows the energy flux $I_{n}$ as a function of $q^{2}$ for the first few bound-state solutions of Eqs. (7). Note that all of these curves have positive curvature over the full range. Figure 2 shows the corresponding bound-state field profiles obtained for $q^{2}=\frac{1}{2}$ and $n=0,1,2$.

\section{STABILITY OF THE BOUND STATES}

In this section we investigate the stability of the bound-state solutions obtained in Sec. II. We concentrate on the higher-bound states $n=1,2$ to illustrate the findings of our study in which we verified our conclusions up to bound states with $n=5$. Section III A describes the numerical stability analysis and the results obtained are discussed in III B. Next, Sec. III C presents an approximate analytical stability theory which reproduces the qualitative features of the numerical results, and therefore reinforces confidence in the numerical results. Direct numerical simulations of the nonlinear wave equation which corroborate our findings are given in Sec. III D.

\section{A. Numerical stability analysis}

To investigate the stability of a general bound-state solution $A_{n}(r)$ with respect to perturbations which break the initial cylindrical symmetry we seek a perturbed solution of the form

$$
A(r, \theta, z)=A_{n}(r)+\mu f(r, z) \cos (m \theta),
$$

where $\mu$ is a small parameter, $f(r, z)$ is a perturbation function, and $m$, an integer, is the azimuthal index. Inserting Eq. (9) into Eq. (5) and linearizing in the small parameter $\mu$ we obtain

$i \frac{\partial f}{\partial z}+\nabla_{r r}^{2} f-\left(q^{2}+\frac{m^{2}}{r^{2}}\right] f+\frac{2 A_{n}^{2} f+A_{n}^{4} f+A_{n}^{2} f^{*}}{\left(1+A_{n}^{2}\right)^{2}}=0$.

This equation for the perturbation function $f$ has many possible types of solution. For our purposes here we specifically want to find those solutions which display exponential growth in the $z$ direction, and which are therefore unstable. Such unstable perturbation eigenmodes propagate with unchanging field profile, although their overall amplitude of course changes, and can be written in the form

$$
f(r, z)=f(r) \exp (\delta z) .
$$

It can be shown that the eigenvalues of this problem are either pure imaginary or pure real. Imaginary eigenvalues correspond to neutrally stable solutions which do not grow upon propagation. In contrast, real positive eigenvalues signal instability since they correspond to ex- ponential growth of the perturbation eigenmode.

The general problem of finding the perturbation eigenmodes of Eq. (10) of the form given in Eq. (11) is a formidable task even computationally. This approach was taken by Kolokolov and Sykov [9], but we have found that some of their conclusions are erroneous. Instead of solving this eigenproblem directly we have used the method of obtaining perturbation eigenmodes and eigenvalues described in Ref. [18]. Namely, for a given $A_{n}(r)$ and $m$, we have solved Eq. (10) with a general nonzero initial condition $f(r, 0)$ using a Crank-Nicholson scheme [19], while following the evolution of the subsequent field profile and calculating the quantity

$$
\begin{aligned}
\delta_{n m}= & (\ln \{\operatorname{Re}[f(r, z+\Delta z)]\} \\
& -\ln \{\operatorname{Re}[f(r, z)]\}) / \Delta z,
\end{aligned}
$$

at each step of the numerical simulation, where $\Delta z$ is the small numerical step length. Clearly, if only one perturbation eigenmode were present at the input then $\delta_{n m}$ would simply be the corresponding eigenvalue by definition (in the limit $\Delta z \rightarrow 0$ ). In general, the input $f(r, 0)$ is composed of several perturbation eigenmodes. However, for large propagation distances that perturbation eigenmode with the largest growth rate $\delta_{n m}$ will dominate since the growth is exponential. We therefore numerically determine the propagation distance beyond which the calculated value of $\delta_{n m}$ does not change perceptibly. From this propagation distance onwards we find that the perturbation eigenmode $f_{n m}(r)$ corresponding to the dominant eigenvalue $\delta_{n m}$ does not change in its field profile. This behavior was verified for both the real and imaginary parts of the dominant perturbation eigenmode. In this way we numerically determine the perturbation eigenmode $f_{n m}(r)$ and corresponding eigenvalue $\delta_{n m}$ for a given bound state $A_{n}(r)$ and azimuthal index $m$ [18]. We expect that the symmetry of the perturbation eigenmode of largest growth rate will dominate the subsequent evolution of a bound state as it goes unstable due to random imperfections or fluctuations in the system. That is, for example, if $m=3$ has the largest growth rate we may expect that the initial cylindrical symmetry is broken to a threefold symmetry, at least during the first stages of propagation.

\section{B. Results from the numerical analysis}

For the saturable nonlinearity considered here it has previously been shown that the ground state is stable. Vakhitov and Kolokolov [7] have shown that a sufficient condition for the stability of the ground state $(n=0)$ is

$$
\frac{\partial I_{0}}{\partial q^{2}}>0
$$

With reference to Fig. 1 we see that the ground state is therefore stable. Consistent with this prediction our numerical stability analysis was unable to find any unstable perturbation eigenmodes for the ground state. In addition, for all higher-bound states considered (up to $n=5$ ), the perturbation eigenmodes with azimuthal indices $m=0,1$ were found to have zero growth rate. The $m=0$ 
perturbation eigenmode corresponds to an instability which preserves the cylindrical symmetry of the problem that causes longitudinal modulations, the "neck-type" instability, whereas the $m=1$ perturbation eigenmode is responsible for instabilities in which the nonlinear wave bends in the transverse direction, the so-called "snaketype" instability [20]. We therefore conclude that the higher-bound states do not display neck-type and snaketype instabilities. However, we remark that, as shown by Zakharov and Rubenchik [20], the inclusion of group velocity dispersion in this problem can lead to the growth of both types of instability.

Figure 3 shows the numerically calculated growth rates $\delta_{n m}$ as a function of scaled effective index $q^{2}$ for the first higher-bound state $n=1$, and azimuthal indices $m=2,3,4,5$. For this case no unstable perturbation eigenmodes were found with azimuthal index larger than $m=5$. Several features are evident from these curves, namely, (1) the $n=1$ bound state is unstable for all values of $q^{2}$ and all the exponentially increasing perturbations depend on the azimuthal angular variable $\theta$, (2) the growth rate is bounded by $\frac{1}{4}$, (3) the maximum value of the growth rate occurs for a finite value of azimuthal index $m$, and (4) the growth rate curves for different values of $m$ cross at certain values of $q^{2}$. In particular, with regard to features (3) and (4), for $q^{2}<0.6$ the perturbation eigenmode with $m=4$ has the largest growth rate, whereas for $q^{2}>0.6$ that with $m=3$ has the largest growth rate (Fig. 3 ). In both cases the most unstable perturbation eigenmode does not correspond to the largest allowed value of $m$, since for $q^{2}<0.6$ the highest growth rate occurs for $m=4$ whereas $m=5$ is also present, and for $q^{2}>0.6$ the highest growth rate occurs for $m=3$ whereas $m=4$ is also present. This result is in contradiction to the findings of Kolokolov and Sykov [9], who concluded that, for nonlinearities for which the ground state is stable, the perturbation eigenmode of largest growth corresponds to the maximum possible azimuthal index $m$. These authors did, however, also find that the growth

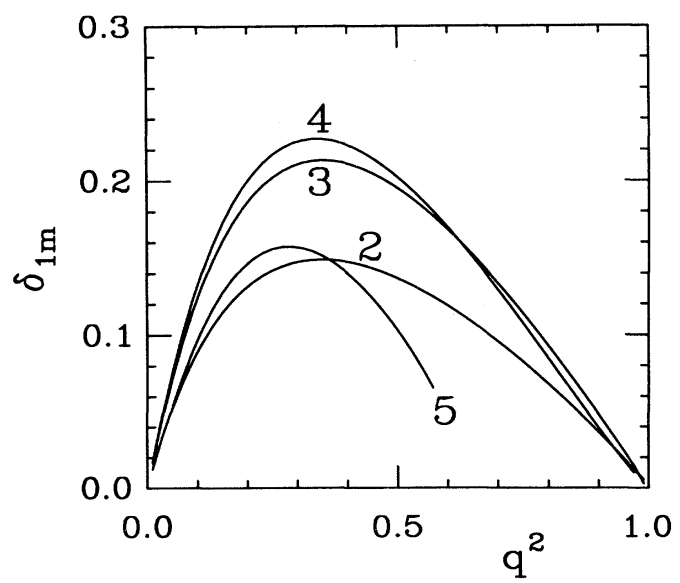

FIG. 3. Growth rates $\delta_{n m}$ of the predominant perturbation eigenmodes with $m$-fold symmetry, associated with the stationary solution for $n=1$, as a function of $q^{2}$.

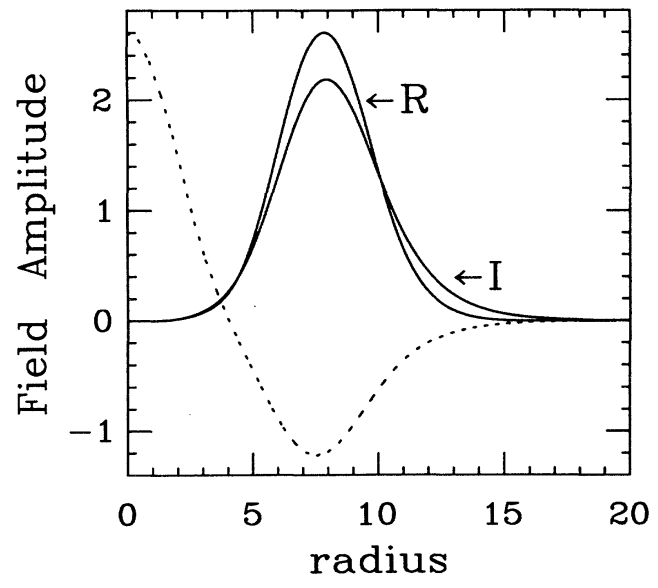

FIG. 4. Real $(R)$ and imaginary $(I)$ part of the perturbation eigenmode with $m=4$ associated with the stationary solution (dashed line) for $N=1$ and $q^{2}=0.35$.

rate was bounded by $\frac{1}{4}$. A qualitative reason for this bound shall be given in the next section.

A typical example of the calculated perturbation eigenmode for $n=1$ is shown in Fig. 4 for $q^{2}=0.35$, and $m=4$. The calculated $n=1$ bound-state profile is shown as a dashed line, and both the real $(R)$ and imaginary $(I)$ parts of the perturbation eigenmode are displayed. Note that the perturbation eigenmode is concentrated mainly around the peak of the annular ring of the bound-state solution.

Figure 5 shows the numerically calculated growth rates $\delta_{n m}$ as a function of scaled azimuthal index $m$ for the second higher-bound state $n=2$ and $q^{2}=0.45$. The numerical results are shown as dots whereas the solid lines

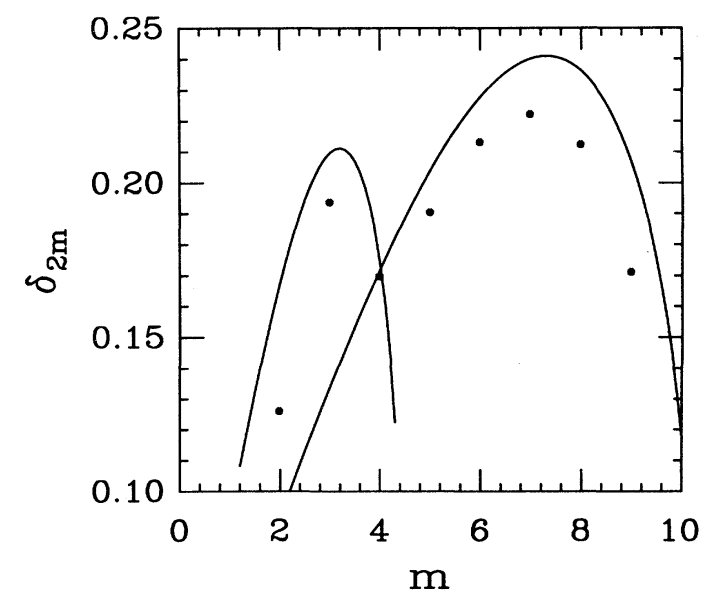

FIG. 5. Growth rates $\delta_{n m}$ of the perturbation eigenmodes with $m$-fold symmetry for $n=2$ and $q^{2}=0.45$. The dots represent the numerically obtained values, while the continuous lines are calculated using Eq. (19), in which we consider $m$ to be a continuous variable. 
are those from an approximate analytic theory to be presented in Sec. III C. Here we clearly see that there are two growth rate peaks in comparison to the case $n=1$ where there is only one. The reason for this is that the second higher-bound state has two annular rings around a central peak. For $m \leq 4$ the unstable perturbation eigenmodes are concentrated mainly around the inner ring, whereas for $m>4$ they are concentrated mainly around the outer ring. This is illustrated in Fig. 6 where we show the unstable perturbation eigenmodes corresponding to the peaks in Fig. 5 for (a) $m=3$, and (b) $m=7$. The calculated $n=2$ bound-state profile is shown as a dashed line for reference, and both the real $(R)$ and imaginary $(I)$ parts of the perturbation eigenmodes are displayed. Therefore, in a sense, each annular ring gives rise to a series of unstable perturbation eigenmodes which have a growth rate structure similar to that for the $n=1$ bound state (see Fig. 3), but with the largest growth rate occurring for different values of azimuthal index $m$ for the two rings.

Our numerical calculations have shown that the same line of reasoning can be applied to the higher-bound
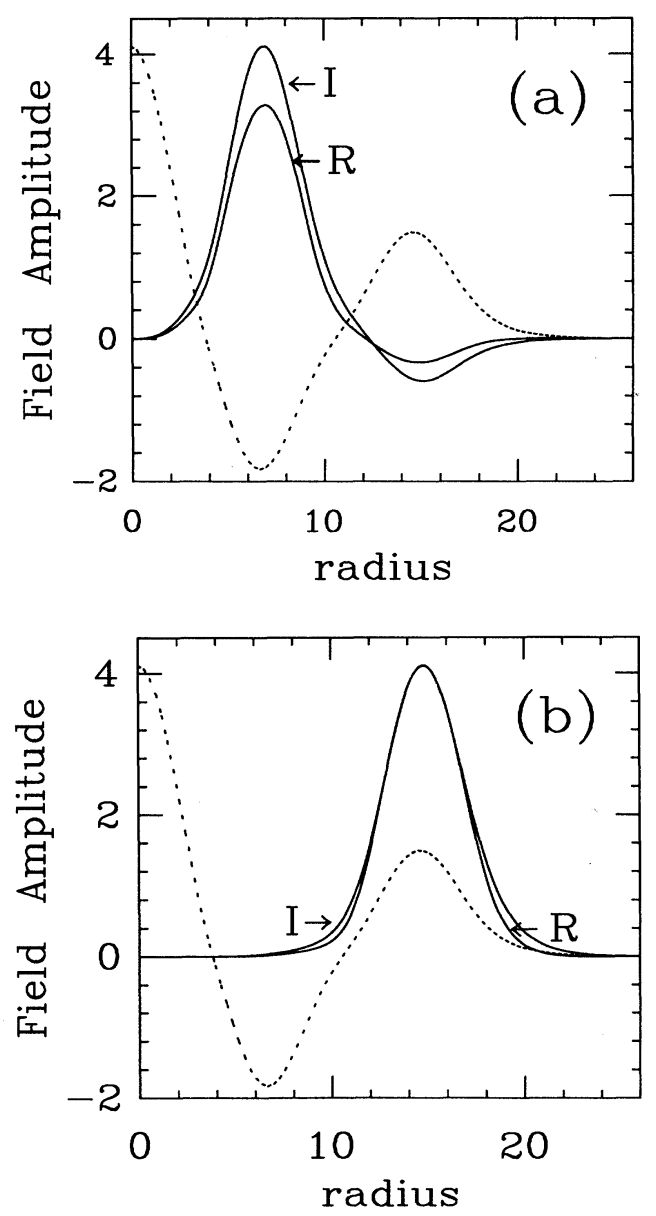

FIG. 6. Same as in Fig. 4 for $n=2$ and $q^{2}=0.45$. (a) $m=3$; (b) $m=7$. states. That is, for the $n$th bound state there are $n$ local maxima in the plot of $\delta_{n m}$ versus azimuthal index $m$ for $q^{2}$ fixed, each maximum being associated with a different annular ring. In this case we expect that as the $n$th higher-bound state loses stability due to random imperfections or fluctuations in the system, the breaking of azimuthal symmetry will be different in each annular ring, at least during the first stages of propagation. In Sec. III D we present direct numerical simulations of the nonlinear wave equation which verify this expectation for the example of the second higher-bound state.

\section{Approximate analytical stability theory}

At present there exists no rigorous stability theory for the higher-bound states. In this section we present an approximate analytic stability theory which yields good qualitative agreement with the numerical results of Sec. III $B$ and lends support to our conclusions based on those results. It also demonstrates that the breakup of the higher-bound states is indeed due to a transverse instability.

To investigate the stability of the higher-bound states we concentrate on the $l$ th annular ring of the $n$th bound state (in general, $l$ may assume values from 1 to $n$ ). Although this annular ring has a field profile we assume that it can be amply characterized by the mean radius $\bar{r}_{n l}$ and amplitude $\bar{A}_{n l}$ given by

$$
\bar{r}_{n l}=\frac{\left[\int_{r_{l}}^{r_{l+1}} 2 \pi r^{2} A_{n}^{2}(r) d r\right]}{\left[\int_{r_{l}}^{r_{l+1}} 2 \pi r A_{n}^{2}(r) d r\right]},
$$

and

$$
\bar{A}_{n l}=\frac{\left[\int_{r_{l}}^{r_{l+1}} 2 \pi r A_{n}^{3}(r) d r\right]}{\left[\int_{r_{l}}^{r_{l+1}} 2 \pi r A_{n}^{2}(r) d r\right]},
$$

where $r_{j}$ is the $j$ th zero of the $n$th bound state, $A_{n}\left(r_{j}\right)=0$. Clearly these quantities measure the mean radius and field amplitude averaged over the annular ring. We have seen in the preceding sections that the unstable perturbation eigenmodes for the higher-bound states are concentrated mainly around the annular rings of these solutions (see Figs. 4 and 6), and one may therefore anticipate that the stability properties are related in some way to $\bar{r}_{n l}$ and $\bar{A}_{n l}$ as defined above.

We now assume that the stability properties of the bound state can be obtained from a simple onedimensional model in which the ring of radius $\bar{r}_{n l}$ is unwound. The equation for the field amplitude $A(s, z)$ along the ring is taken of the form

$$
i \frac{\partial A}{\partial z}+\frac{\partial^{2} A}{\partial s^{2}}-q^{2} A+\frac{|A|^{2} A}{1+|A|^{2}}=0,
$$

where $s$ is the path length along the ring, and

$$
q^{2}=\frac{\bar{A}_{n l}^{2}}{1+\bar{A}_{n l}^{2}} .
$$


We have chosen this value of the effective index $q^{2}$ so that $A=\bar{A}_{n l}$ is a stationary solution of Eq. (16). Notice that in this picture the bound-state solution is simply represented by the setting $A=\bar{A}_{n l}$ at all points around the ring. We investigate the stability of this solution by setting

$$
A(s, z)=\bar{A}_{n l}+\mu \cos (m \theta) \exp \left(\delta_{n l m} z\right),
$$

where we have used $s=\bar{r}_{n l} \theta / 2 \pi$ in Eq. (16), $m$ is the azimuthal index, $\mu$ is a small constant, and $\delta_{n l m}$ is the growth rate. Note that the form chosen for the perturbation in Eq. (18) ensures that the field is periodic in $\theta$, but allows for modulations to develop around the ring. This means that we are dealing with a transverse or modulation instability which breaks the azimuthal symmetry of the problem [11, 14]. By substituting Eq. (18) into (16), linearizing with respect to $\mu$, and solving for $\delta_{n l m}$ we obtain

$$
\delta_{n l m}=\left[\frac{m}{\bar{r}_{n l}}\right]\left[\frac{2 \bar{A}_{n l}^{2}}{\left(1+\bar{A}_{n l}^{2}\right)^{2}}-\left(\frac{m}{\bar{r}_{n l}}\right]^{2}\right]^{1 / 2} .
$$

This approximate solution for the instability growth rate neglects the transverse structure of the field and must therefore be judged by the quality of the results it produces in comparison to the numerical solutions. Figure 7 shows the results obtained from Eq. (19) corresponding to the numerical solutions given in Fig. 3. Excellent qualitative agreement is clearly seen, though Eq. (19) overestimates the actual growth rate. Note, in particular, that the approximate solution correctly predicts the crossing in growth rate between the curves for $m=4$ and 3 which occurs at $q^{2} \cong 0.6$, and it also correctly predicts that there are no unstable eigenmodes with $m>5$ for $n=1$. In addition, it is simple to show that all (real and positive) growth rates $\delta_{n l m}$ predicted by Eq. (19) are bounded by $\frac{1}{4}$ in agreement with the numerical calculations.

As a second example we consider the second higher-

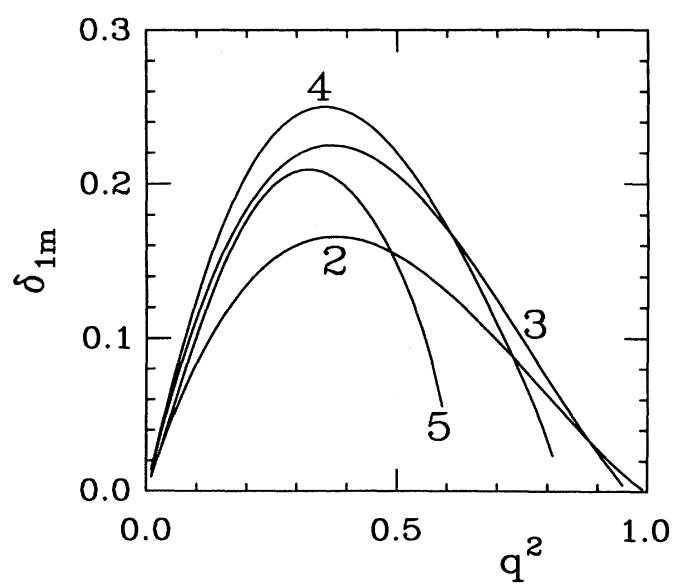

FIG. 7. Same as in Fig. 3 but for $\delta_{n m}$ obtained from Eq. (19). bound mode $n=2$ which has two annular rings. The solid curves in Fig. 5 show the calculated growth rates as a function of $m$ for $q^{2}=0.45$, and the dots represent the numerical results. (The analytic results are shown as solid lines but only the integer values of $m$ are physically relevant for this problem.) Once again the overall qualitative agreement is very good; in particular, the approximate analytic theory correctly predicts the maxima at $m=3,7$.

We note here that the exact form of the model chosen for the nonlinearity does not affect the results qualitatively, as long as it obeys two conditions: (1) the slope of the
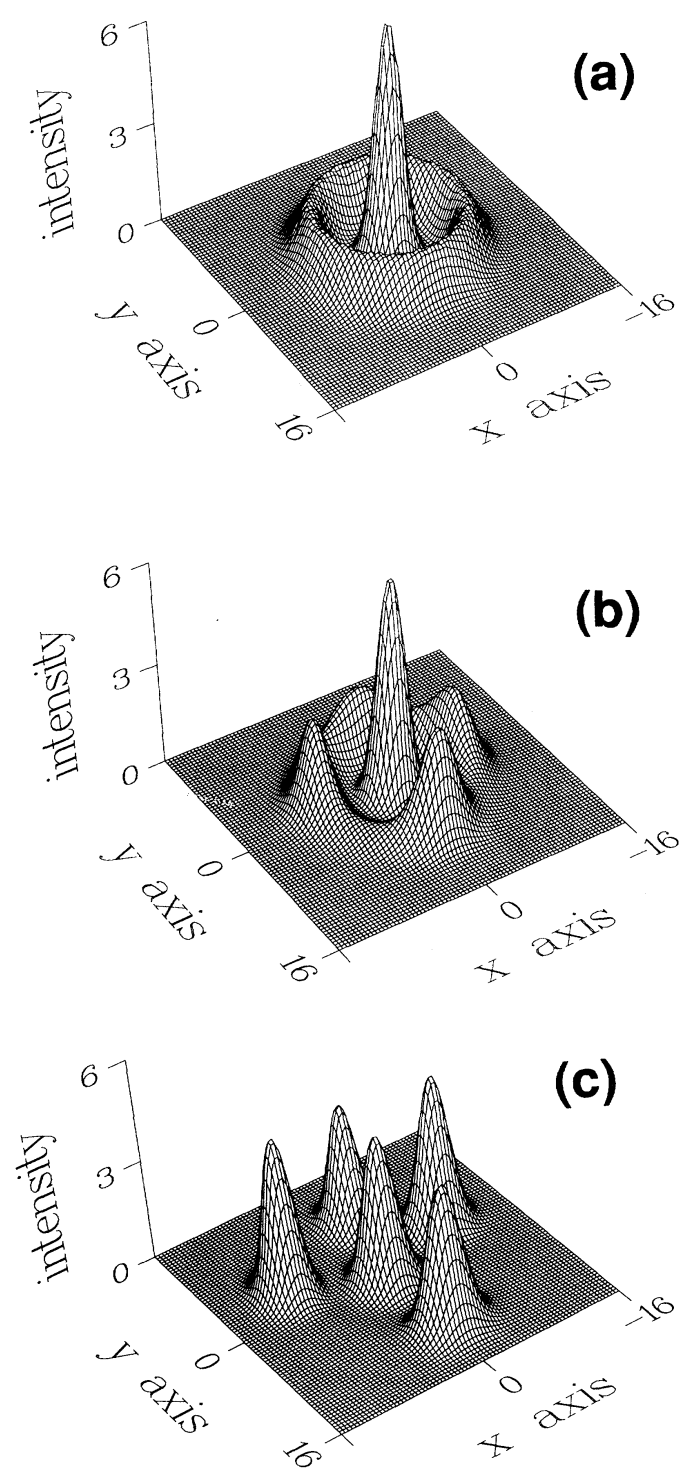

FIG. 8. Spatial distribution of the intensity at different propagation distances $z$. (a) Initial input beam $(z=0)$, which corresponds to the slightly perturbed stationary bound state for $n=1$ and $q^{2}=0.35$; (b) $z=60$; (c) $z=80$. 
dielectric function $\epsilon\left(|E|^{2}\right)$ is positive for all finite $|E|^{2}$, and (2) the nonlinearity saturates to some finite value $\Delta \epsilon_{\text {sat }}$. Condition (2) frustrates the catastrophic selffilamentation which can occur for self-focusing Kerr media in two dimensions (2D). Condition (1) ensures that when the response if linearized with respect to a small change in the field $\mu$ [as in Eqs. (18) and (19)], the term proportional to $\mu$ will always be positive, and the growth rate will be of the form of Eq. (19). Our particular choice of model was motivated by its basis in simple physical systems; the well-studied "two-level atom" response of a system to an optical field yields Eq. (1) (see, for example, Yariv [21]).

\section{Numerical simulations}

In this section we present some numerical simulations of the nonlinear wave equation (5) which corroborate the
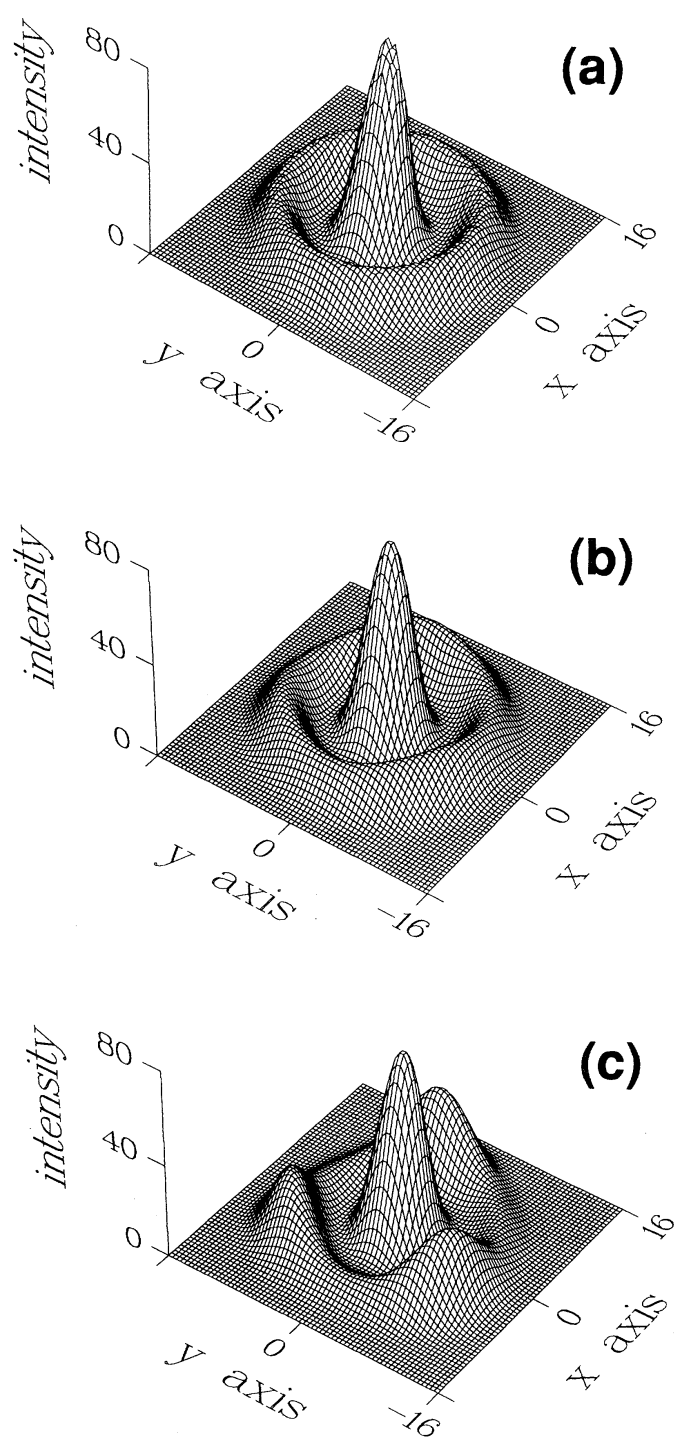

FIG. 9. Same as in Fig. 8 for $n=1$ and $q^{2}=0.8$. (a) $z=0$; (b) $z=140$; (c) $z=170$. findings of our stability analysis. In particular, we have solved Eq. (5) using the split-step method [22] on a Cartesian grid with the initial condition

$$
A(x, y, 0)=A_{n}(r)[1+\Gamma(x, y)],
$$

where $\Gamma$ is a random noise term obeying

$$
\langle\Gamma(x, y)\rangle=0, \quad\left[\left\langle|\Gamma(x, y)|^{2}\right\rangle\right]^{1 / 2}=\frac{1}{100} .
$$

The noise term is intended to mimic random fluctuations in the input beam which will serve to seed any latent instability in the system. All of the numerical simulations reported here were verified for several realizations of the noise term to ensure that our conclusions were not the product of a wild fluctuation which biased one specific perturbation eigenmode.
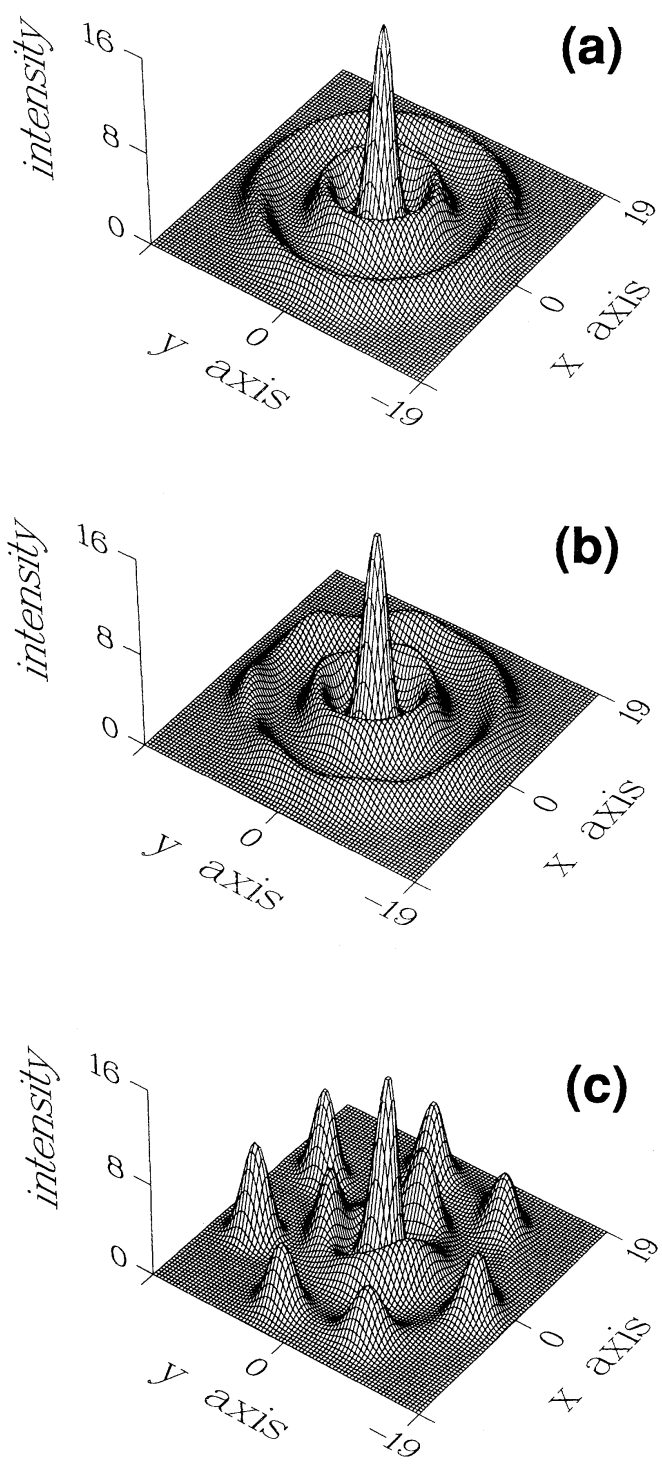

FIG. 10. Same as in Fig. 8 for $n=2$ and $q^{2}=0.45$. (a) $z=0$; (b) $z=50$; (c) $z=70$. 
Figure 8 shows the evolution of the initial field profile for the example represented in Fig. 4, where $n=1$ and the perturbation eigenmode with $m=4$ has largest growth rate. As expected, the field profile develops an azimuthal modulation which has four-fold symmetry as it propagates. In contrast, Fig. 9 shows the corresponding results for $q^{2}=0.8$, for which $m=3$ has the largest growth rate, and an azimuthal modulation develops which has threefold symmetry. With reference to both Figs. 3 and 7, we see that these results are in complete agreement with both the numerical and analytic predictions.

As a further demonstration of the validity of our analyses we consider the second higher-order bound state with $n=2$. According to the results leading to Fig. 5, for $q^{2}=0.45$ the perturbation eigenmode with largest growth rate occurs for $m=3$ for the inner annular ring $(l=1)$, and $m=7$ for the outer annular ring $(l=2)$. Figure 10 shows the evolution of the initial field profile for this case and as predicted the inner ring develops an azimuthal modulation with threefold symmetry, whereas the outer ring develops an azimuthal modulation with sevenfold symmetry. Again the results are in complete agreement with both the numerical and analytic predictions. We remark that such agreement between the numerical simulations and analytic predictions was found in all the cases we examined within the range $0<q^{2}<1$.

Upon further propagation each of the numerical simulations reported here lead to the formation of filaments or hot spots. The number of filaments formed was accurately predicted as the azimuthal index of the perturbation eigenmode of largest growth rate, as expected. However, the dynamics of the filaments as they propagate was generally very complex, and is beyond the scope of the present paper.

\section{SUMMARY AND CONCLUSIONS}

In this paper we have presented a combined numerical-theoretical investigation of the stability of the cylindrically symmetric higher-bound states which can be formed in a self-focusing medium with saturation. The higher-bound states were shown to display transverse instabilities which break the azimuthal symmetry of the problem. An approximate analytic theory was presented which gives excellent qualitative agreement with the numerically calculated instability growth rates.

We anticipate that these results will be of more general utility in the analysis of the beam breakup and filamentation which occurs for nonstationary solutions. For example, Konno and Suzuki have shown numerically that beam breakup of an initially broad Gaussian beam occurs only after the beam has undergone significant reshaping and formed an annular ring, whereupon breakup into four filaments occurs [15]. This is highly suggestive of the same phenomena reported here for the first higherbound state. In recent work Heatley, Wright, and Stegeman have shown that when spatial rings are emitted into a nonlinear cladding medium bounding a linear fiber core beam breakup can occur [23]. The application of the approximate analytic model to such nonstationary cases will be reported in a future publication.

\section{ACKNOWLEDGMENTS}

This work was supported by the Army Research Office (Grant No. DAAL03-88-K0066) and the Joint Services Optical Program. J. M. Soto-Crespo acknowledges financial support from the Ministerio de Educacion y Ciencia of Spain. Computer time from the Air Force SuperComputer Center at Kirtland is gratefully acknowledged.
*Jointly with the Physics Department, University of Arizona.

[1] R. Y. Chiao, E. Garmire, and C. H. Townes, Phys. Rev. Lett. 13, 479 (1964).

[2] P. Kelley, Phys. Rev. Lett. 15, 1005 (1965).

[3] For a comprehensive review of experimental work on self-focusing and self-trapping see Y. R. Shen, Prog. Quantum Electron. 4, 1 (1975).

[4] For a comprehensive review of theoretical work on selffocusing and self-trapping see J. H. Marburger, Prog. Quantum Electron. 4, 35 (1975).

[5] Z. K. Yankauskas, Izv. Vyssh. Uchebn. Zaved. Radiofiz. 9, 412 (1966) [Sov. Radiophys. 9, 261 (1966)].

[6] H. A. Haus, Appl. Phys. Lett. 8, 128 (1966).

[7] N. G. Vakhitov and A. A. Kolokolov, Izv. Vyssh. Uchebn. Zaved. Radiofiz. 16, 1020 (1973) [Sov. Radiophys. 9, 261 (1966)]; A. A. Kolokolov, ibid. 17, 1332 (1974) [ibid. 17, 1016 (1976)].

[8] For a review of self-focusing instabilities see, e.g., J. J. Rasmussen and K. Rypdal, Phys. Scr. 33, 481 (1986), and references therein.

[9] A. A. Kolokov and A. I. Sykov, Zh. Prikl. Mekh. Tekh.
Fiz. 4, 55 (1975) [J. Appl. Mech. Tech. Phys. 4, 519 (1975)].

[10] J. A. Fleck and C. Layne, Appl. Phys. Lett. 22, 467 (1973).

[11] A. J. Campillo, S. L. Shapiro, and B. R. Suydam, Appl. Phys. Lett. 23, 628 91973).

[12] A. J. Campillo, S. L. Shapiro, and B. R. Suydam, Appl. Phys. Lett. 24, 178 (1974).

[13] J. E. Bjorkholm and A. Ashkin, Phys. Rev. Lett. 32, 129 (1974).

[14] B. R. Suydam, IEEE J. Quantum Electron. QE-11, 225 (1975).

[15] K. Konno and H. Suzuki, Phys. Scr. 20, 382 (1979).

[16] M. D. Feit and J. A. Fleck, J. Opt. Soc. Am. B 5, 633 (1988).

[17] J. H. Marburger and E. Dawes, Phys. Rev. Lett. 21, 556 (1968); Phys. Rev. 179, 862 (1969).

[18] N. N. Akhmediev, V. I. Korneev, and Yu. V. Kuz'menko, Zh. Eksp. Teor. Fiz. 88, 107 (1985) [Sov. Phys._JETP 61, 62 (1985)].

[19] W. H. Press, B. P. Flannery, S. A. Teukolsky, and W. T. Vetterling, Numerical Recipes (Cambridge University Press, Cambridge, England, 1986), p. 640. 
[20] V. E. Zakharov and A. M. Rubenchik, Zh. Eksp. Teor. Fiz. 65, 997 (1973) [Sov. Phys. - JETP 38, 494 (1974)].

[21] A. Yariv, Quantum Electronics, 3rd ed. (Wiley, New York, 1989), pp. $155-161$.

[22] J. A. Fleck, J. R. Morris, and M. D. Feit, J. Appl. Phys.
10, 129 (1975); M. D. Feit and J. A. Fleck, J. Opt. Soc. Am. B 5, 633 (1988).

[23] D. R. Heatley, E. M. Wright, and G. I. Stegeman, Opt. Lett. 16, 291 (1991). 